\title{
RESEARCH ON MOBILE USER INTERFACE FOR ROBOT ARM REMOTE CONTROL IN INDUSTRIAL APPLICATION
}

\author{
JIANGNAN NI*AND VIPIN BALYAN †
}

\begin{abstract}
The mobile interfaced robot arms are majorly being used nowadays in order to provide the remote-control applicability for various industrial and manufacturing applications. This article proposes a robotic arm platform for controlling the industrial application. The proposed system includes various modules like a robot arm, a controller module and a remote mobile operating application for visualizing the robot arm angles having real time applicability. Augmented reality (AR) is utilized for robot control WIFI communication and the robot angle information is obtained for varying real time environment. This novel approach incorporated the AR technology into mobile application which allow the real time virtual coordination with physical platform. The feasible trajectories are generated using the proposed methodology and a comparison is made between the desired and real trajectory paths. The simulation results are obtained for various assessment indicators and effectual outcomes are achieved with $98.03 \%$ accuracy value and $0.185,0.180$ of error and loss values for training phase. The accuracy value of $97.65 \%$ is achieved for testing phase with corresponding 0.209 and 0.190 minimum error and loss values. The proposed platform provides the feasible and reliable outcomes in the real time environment for real time manufacturing industry applications.
\end{abstract}

Key words: Mobile interface; Augmented reality; real time environment; trajectory path; virtual coordination; manufacturing industry application.

AMS subject classifications. 65D19

1. Introduction. The advent of widespread utilization of wireless technology for mobile communication is now being used very often in the industrial automation and many other evolving sectors. The mobile systems are being used nowadays for the automation and mechanical movement [1]. There are several applications of industrial automation in various sectors like surveillance in army [2-4], different automation industry and petroleum, oil and other industrial sectors [5-7]. The various application of robotics in different industrial sectors indicates that the automobile industry plays a significantly leading role in the employment of robots [8]. The clear representation of various sectors utilizing the robotics are depicted in Figure 1.1.

The other sectors utilizing the robotics in day-to-day application are automatic surveillance systems initiated for the safety of mankind, gas monitoring and oil pipeline systems for controlling extremely high pressure [9]. For this purpose, small robots are employed which can easily fit inside the pipelines and move around in order to monitor the pipeline issues [10]. For the automobile industry, robots are used in the inventories for manufacturing purpose and also at the assembly line in order to obtain high accuracy and speedy manufacturing. Utilization of the modern wireless technology for the operation and control of manufacturing process make use of mobile devices for sending the commands to machine using a computer or a mobile system [11].

The robot systems used in the past few decades involve the robot arms or hand manipulators for the controlling purpose. The current act of robot arm controller involves the human specialists. These specialists train and coordinates the controller's developments for leading diverse pre-characterized tasks. The significant learning includes the changing of robot arm joint position, when any impediment is experienced by the controller during the pre-planned task [12-14]. This learning method is reasonable for a particular assignment and specific climate, yet as the work space changes, this kind of manual learning system fails. At that point, the robot learning should be done again for an alternate workplace or the dynamic environment. The other limitation of present methodologies in the robot arm controller is its optimality and undertaking adequacy. The manual

\footnotetext{
*Henan Polytechnic Institute, Nanyang 473000, China (jiangnanni531@gmail.com).

${ }^{\dagger}$ Department of Electrical, Electronics and Computer Engineering, Cape Peninsula University of Technology, Cape Town, South Africa (vipin.balyan@rediffmail.com).
} 


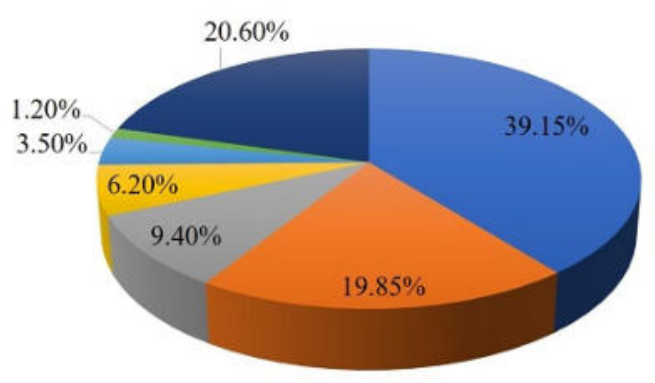

- Automobile Industry

= Electrical/Electronic Industry

w Metals and Machinery

Industry

in Chemical, Rubber \& Plastic

Industry

= Food and Beverage Industry

- Pharpahceutical and

Cosmetic Industry

a Other Industry

FIG. 1.1. Industrial Applications of Robotics

learning is task explicit, accordingly, tracking down an ideal way for robot controller in the dynamic environment is troublesome $[15,16]$. With the new progression in profound learning, neural networks and reinforced learning mechanisms are being utilized by the researchers for the learning interaction in different scenarios $[17,18]$.

This article proposes a robotic arm platform for controlling the industrial application using the manipulation concept. The presented system comprises different modules like the robot arm, a controller module and a mobile operating application for visualizing the robot arm angles in real time scenario. This work focuses on the utilization of augmented reality (AR) application for robot control utilizing the WIFI communication channel. It contributes in obtaining the robot angle information using AR application in the real time scenario. This work specifically contributes in manufacturing industry, information technology and various other application platforms. The novelty of this work likes in the utilization of augmented reality into the mobile application, thus allowing the remote access of real time virtual coordination with the physical platform. The reference profile is created for programming the robot using a desktop application and the controller module is utilized for providing trajectory path to the joint movement of the robot arm. For the manufacturing industry application, the proposed platform provides the feasible and reliable outcomes in the real time environment.

The rest of this article is structured as: literature survey is presented in section 2 which is trailed by the methods and materials described in section 3. Section 4 presents the experimental results and analysis which is further trailed by the concluding remarks of the research work in section 5 .

2. Literature Review. The utilization of robotics for the various applications in networking and various other industrial sectors originated from the concept of tele-robotics. Tele-robotics have been evolved with the development of internet and networking expertise. There are several networking robots developed by the researchers and various innovation have been done in this field [19]. There are several robotic applications which are being utilized for the public usage and exploration [20]. The network robot expansion has evolved the scope for integration in various domains.

There are various applications of robots in medical science field. The literature overview presented in [21] addressed the problem of invasive surgery using augmented reality system. Authors in [22] summarized the robotic systems using the virtual reality technology for the surgical assistance using the robotic platforms. Systems utilized in $[23,24]$ consists of robot-based systems for providing assistance for efficient operation of surgeries for improving the safety of patients, medical manipulations, etc. they utilized the augmented reality-based platforms for the automated detection and diagnosis of the pathological regions. The recent work presented in Quero, et al. [25] dealt with the robotic platforms for achieving high precision for liver surgeries using 3D image visualization. The improvement in the surgeon perception is noticed by the utilization of $3 \mathrm{D}$ visualization method and robotics. Authors in [26] utilized the visual form of x-rays for getting the real time perception of invasive laparoscopic surgery. The robotics platform has been used for the integration of surgery and robotics 


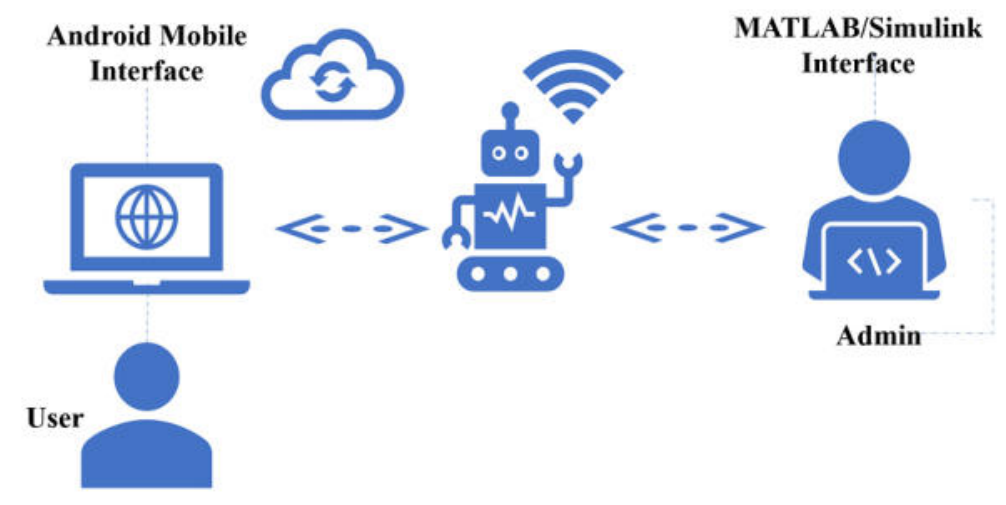

FIG. 3.1. Workflow of the proposed system including various working modules

in the medical scenarios [27]. There are various developments being noticed in recent literary work being done from the last 5 years.

Recently, there are various state of the art work in the field of simulation and design in industrial manufacturing domain. The article presented by Mourtzis [28] implemented a design and simulation platform for the manufacturing process and along with another author Mourtzis and Zogopoulos [29] an augmented reality-based interface is provided for supporting the assembly processing in the manufacturing industry. This industrial advent continued with the integration of robotics along with the warehouse designing in the papermaking industry [30]. For such application robotics plays a very important role in the industrial as well as programming platforms. Such applications of robotics in the industrial sector improves the significance of motion planning as well as robots while reducing the extensive human labor [31]. Ong et al. [32] interfaced the robotics in welding industry scenario for ease of workload reduction. Avalle et al. [33] also presented a scenario in which welding robots are developed for ease of programming and to complete the complex tasks with requirement of human expertise. Gong et al. [34] used the robot for providing technical assistance to the human operators for the reduction of cognitive workload. This can be accomplished by involving the robot-based object manipulation for real-time environment.

3. Material and Methods. This section provides the outline of the proposed system including three major modules like the robot arm, a controller module and a mobile operating application. The robot arm is responsible for the movement and the controller module receives the signal from the application platform and then send it to the robot arm encoder. The mobile operating application proves the real time visualization and remote access of robot angles using the augmented reality (AR) interfacing. The entire workflow of the proposed system is depicted in Figure 3.1 which is further detailed in this section.

- The admin sends a command to the robot arm using a desktop interface and a USB serial protocol.

- The robot arm controller encodes the command and revert back as the angular movements for each of the articulation which are plotted as graphs at the MATLAB/Simlink interface.

- The connection request is sent by the user from the mobile interface using the WIFI communication channel. The control unit of the robot arm accept this request and allow the mobile interface to establish the remote access interaction connection.

- Using this connection, the mobile interface is able to identify the robot arm locations specified by the controller module. The degree of each articulation is identified and displayed on the remote mobile interface.

3.1. Robotic arm and the controller module. The flowchart of robotic arm and the controller module is presented in Figure 3.2. The flowchart indicated in Figure 3.2 establishes a configuration routine using the USB connection for external communication. The connection request is made by the configuration manager and it detects whether any request is made either by the mobile or the desktop interface. The configuration connection requirements are verified and the base angle for the robot is generated. The robot arm angle is 


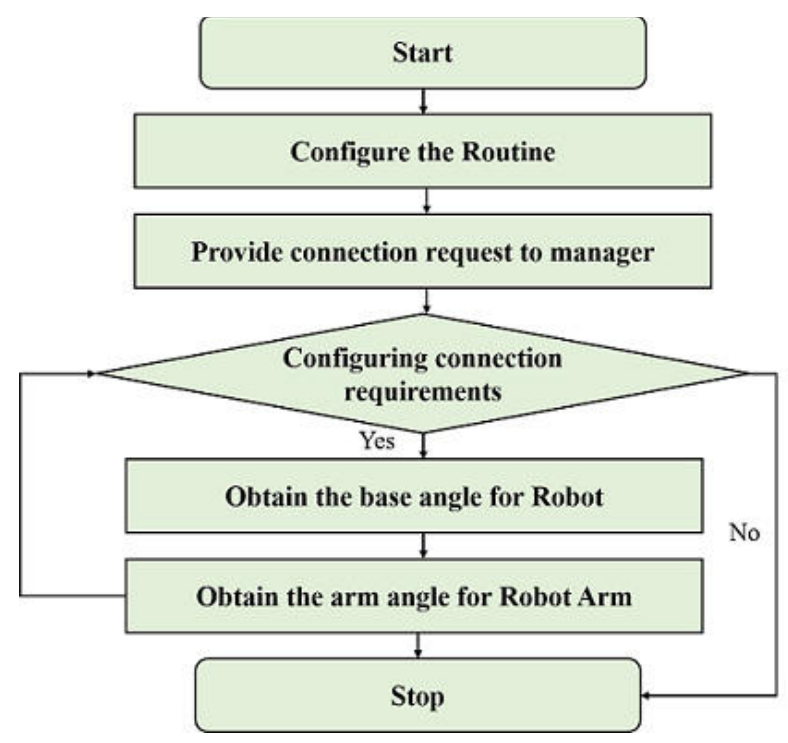

FIG. 3.2. Flowchart of robotic arm and the controller module

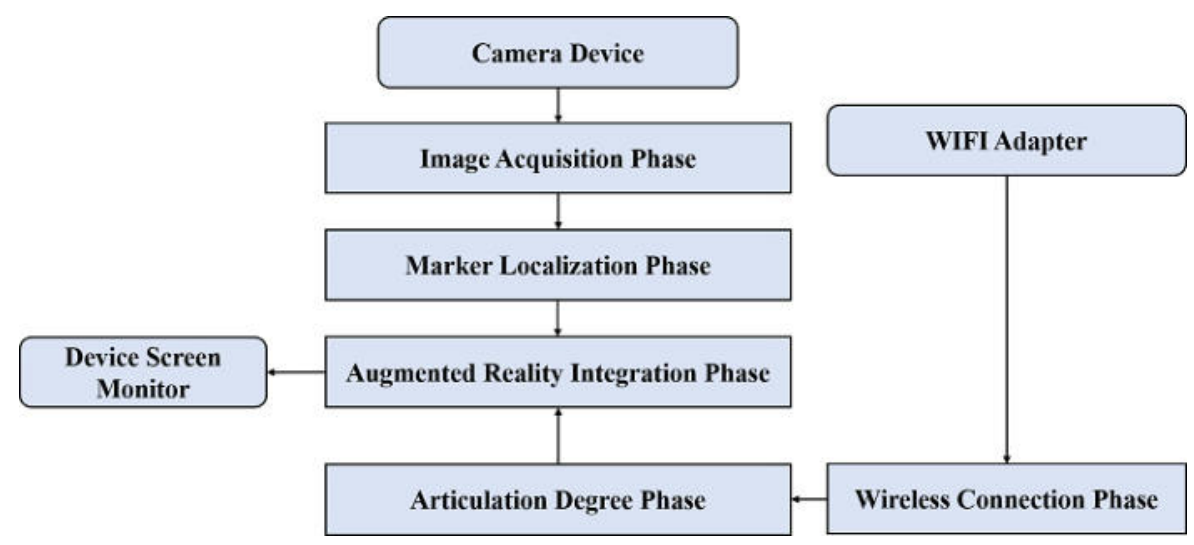

FIG. 3.3. Main phases of mobile interface module

obtained and articulated into digital form and these angle specifications are sent to the mobile interface using the WIFI connectivity.

3.1.1. Mobile Interface Module. The third module of the proposed system consists of the mobile interface whose main phases are depicted in Figure 3.3. The major phases in the mobile interfacing module are as follows:

i. Image Acquisition Phase: This phase acts as a connection in between the physical optical sensor and the application module. The image acquisition phase exploits a camera-based technique for the assessment of robot arm location.

ii. Marker Localization Phase: This phase is responsible for obtaining the robot arm marker locations from the input image or video captured using the image acquisition phase. The obtained image is used in the training phase for obtaining the specific marker locations and these locations are stored in the system so that it can be utilized in the testing phase.

iii. Degree Articulation Phase: It performs the operation of robot arm degree articulation depending upon the marker location defined by the marker localization phase. For each location marked, this phase specifies an angular degree which is to be passed to AR integration phase. 
TABLE 3.1

Assessment Indicators

\begin{tabular}{|l|l|l|}
\hline Indicator & Computation Formula & Eq. no. \\
\hline \hline Sensitivity (SN.) & $S N .=\frac{T P}{T P+F N}$ & $(1)$ \\
\hline Specificity (SP.) & $S P .=\frac{T N}{T N+F P}$ & $(2)$ \\
\hline Precision (PR.) & $P R .=\frac{T P}{T P+F P}$ & $(3)$ \\
\hline F-Measure & $F-M e a s u r e .=\frac{2 * P R . * S N .}{P R .+S N .}$ & $(4)$ \\
\hline Accuracy (ACC.) & $A C C .=\frac{T P+T N}{T P+F N+T N+F P}$ & $(5)$ \\
\hline
\end{tabular}

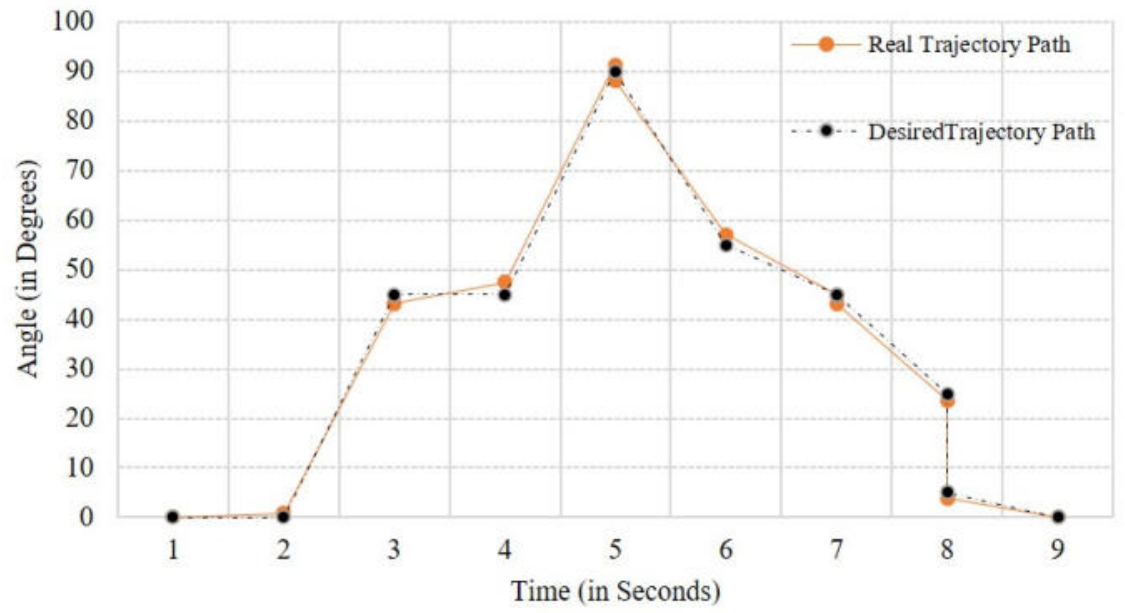

Fig. 4.1. Analysis of real and desired trajectory paths

iv. Wireless Connection Phase: This phase is able to detect the robot compatibility with the WIFI module and it is responsible for linking the robot device with the entire platform. The wireless linkage is able to link the data from the application to the platform and also responsible for activating the rest of the functionalities of the application module.

v. Augmented Reality Integration Phase: This phase obtains the input from the localization marker and degree articulation phase and then generates the projection image which can be seen on the monitor screen of the device.

3.1.2. Assessment Indicators. The confusion matric is created for the desired and the actual class and based on that matric, the behavior of the proposed framework is assessed using the true and false indications of the robot arm path trajectory. The different indicators utilized for this purpose are given in Table 3.1. TP, $\mathrm{TN}, \mathrm{FP}$ and FN indicate the true positive, true negative, false positive and false negatives respectively.

4. Results and Discussion. The experimental set-up comprises of optical encoders with $12 \mathrm{~V}$ DC motor, a WIFI module for communicating to the robot arm using AR application, a robot arm and a mobile interfacing device with a device screen camera at the back of the device. Several experimental tests are performed and comparative analysis is done for validating the trajectory localization and effectiveness of the robot arm platform for various applications.

4.1. Comparative analysis of the robot arm trajectory. A comparison is done using the MATLAB/Simulink environment which plots the graph between the desired and the real path of the robot arm. The movement commands are generated using the desktop interface for every movement of the robot arm and the articulation is processed by the controlling module of the robot arm. The analysis of real and desired trajectory path is done in Figure 4.1. 
TABLE 4.1

Assessment indicator values obtained after experimentation

\begin{tabular}{|l|l|l|}
\hline Performance Indicator & Training & Testing \\
\hline \hline Sensitivity (SN.) & $98.28 \%$ & $97.72 \%$ \\
\hline Specificity (SP.) & $97.54 \%$ & $97.54 \%$ \\
\hline Precision (PR.) & $98.77 \%$ & $98.36 \%$ \\
\hline F-Measure & $96.59 \%$ & $96.59 \%$ \\
\hline Accuracy (ACC.) & $98.03 \%$ & $97.65 \%$ \\
\hline
\end{tabular}

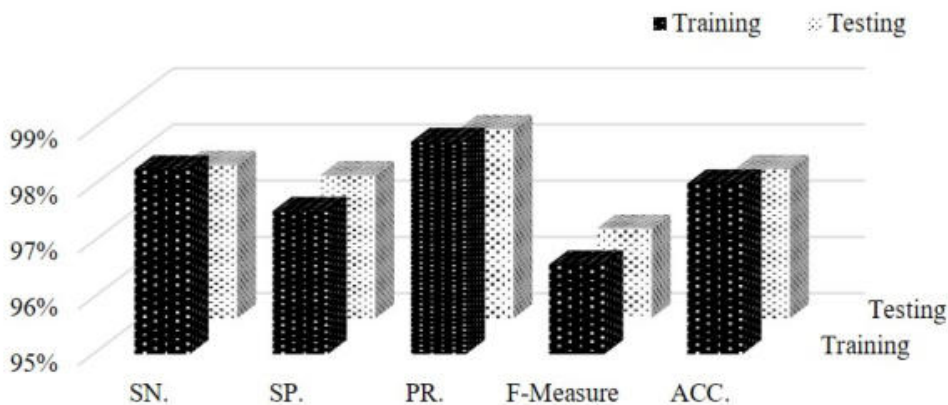

FIG. 4.2. Graphical representation of assessment indicators

The robot arm learning is done using the analysis of real and desired trajectory paths. Further, the planning and control action is determined by the learned path information. This learning is application for trajectory planning in case of unfavorable environment in the testing phase.

The data from training and testing phase are compared in terms of true negative, true positive, false negatives and false positives. On the basis of this information, the confusion matrix is created and the performance is evaluated in terms of various assessment indicators.

4.2. Performance evaluation in terms of assessment indicators. The performance of the proposed robot arm-based platform is assessed in terms of different indicators for both training and testing scenarios. Trained outcomes are stored as learning which are further utilized in the testing phase for dealing with unfavorable environment. The outcomes obtained are shown in Table 4.1 and its graphical depiction is provided in Figure 4.2.

These outcomes reveal the practicability of the proposed robot arm-based system as the SN., SP., PR., Fmeasure and ACC. values of 98.28\%, 97.54\%, 98.77\%, 96.59\% and 98.03\% is achieved for the training phase and $97.72 \%, 97.54 \%, 98.36 \%, 96.59 \%$ and $97.65 \%$ are achieved for the testing phase respectively. The feasible and effective solution is achieved using the robot arm-based solution which is applicable for unfavorable industrial environment. The cost effectiveness is evaluated in terms of error and losses during the system integration and working which are depicted in Figure 4.3 and Figure 4.4 respectively.

The errors indicated in Figure 4.3 provides the depiction of reduction in error with the increasing number of iterations for both the training and testing phases. The minimum error value of 0.209 is achieved for 50 th iteration in the testing phase which is a bit more comparative to the error value of 0.185 obtained for the training phase.

Figure 4.4 reveals that losses also reduces significantly with the increase in the iteration count. The minimum loss value of 0.180 is achieved for the training phase which is comparable to the minimum testing loss of 0.190 without leading to overfitting. 


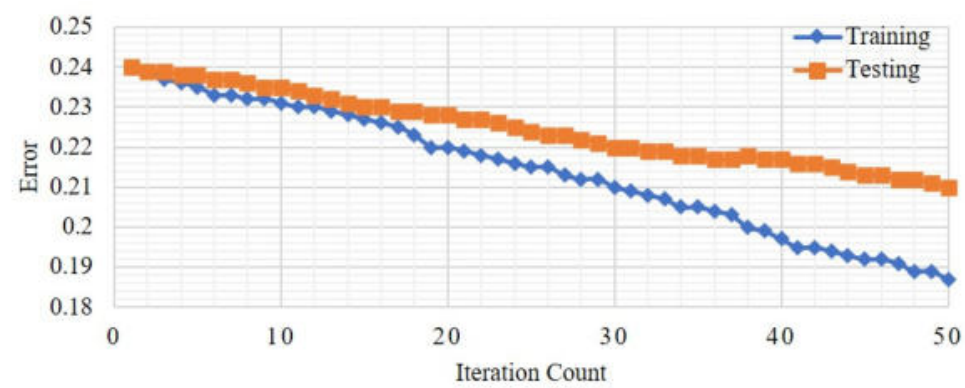

FiG. 4.3. Error Rate outcomes

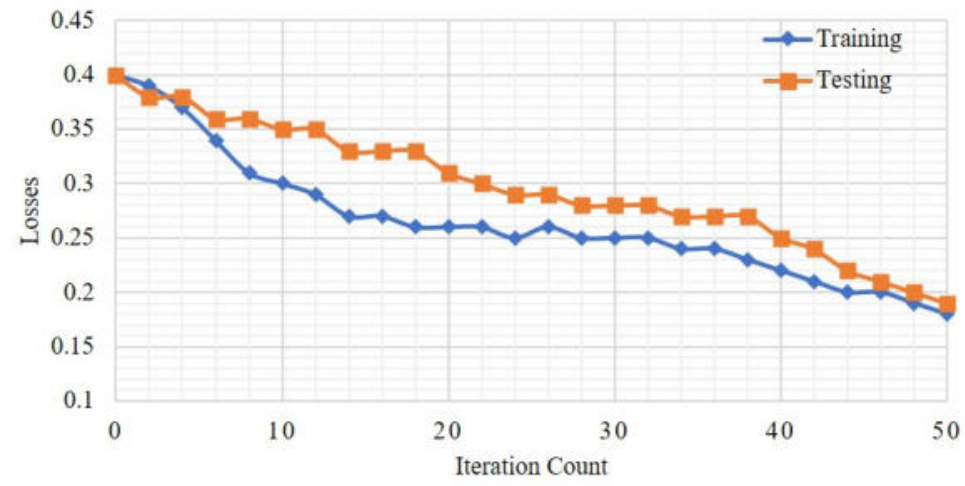

FIG. 4.4. Loss Value outcomes

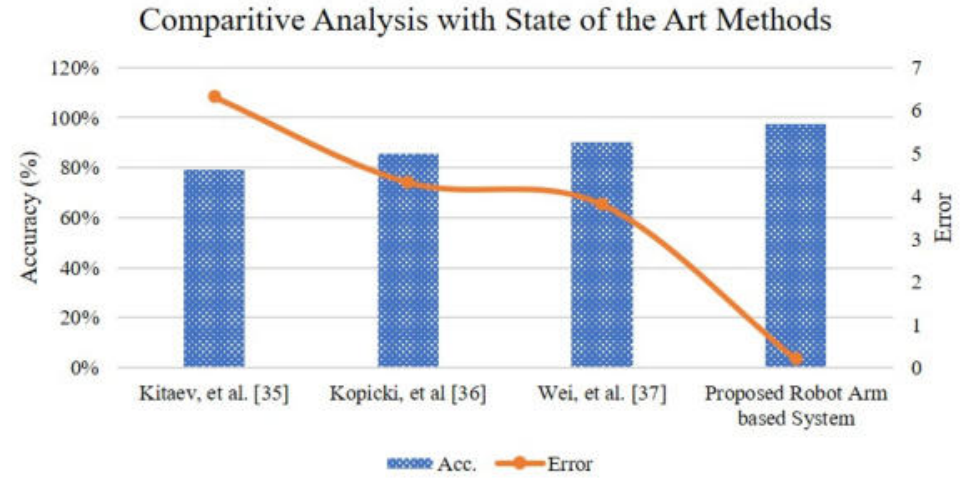

FIG. 4.5. Comparative analysis with current techniques in this domain

4.3. Comparative assessment with the current techniques in this domain. The comparison of the proposed robot-based solution is done with other state of the art research work going on in this field. The comparative study of accuracy and the error is depicted in Figure 4.5.

It is evident from the comparison of the proposed method with state-of-the-art methods that the proposed robot arm-based platform out performs the conventional methods by providing a maximum accuracy percentage improvement of $23.61 \%$ while obtaining an error minimization of 0.209 . This comparative analysis reveals that proposed methodology is competent and reliable for monitoring the control and planning actions for unfavorable industrial applications. 
5. Conclusion. In this work, a robotic arm platform for controlling the industrial application using the manipulation concept is proposed that utilizes the augmented reality (AR) application for robot control. The major components of the proposed system are robot arm, a controller module and a mobile operating application for robot visualization in real time environment. The feasible task-specific trajectories are generated using the proposed methodology and a comparison is made between the desired and real trajectory paths. The simulation results are observed using various assessment indicators which provides effectual outcomes for all the different environmental scenarios. The training phase provides the values of $98.28 \%, 97.54 \%, 98.77 \%, 96.59 \%$ and $98.03 \%$ for sensitivity, specificity, precision, f-measure and accuracy, respectively, while maintaining a reliable error rate of 0.185 with 0.180 loss value. Similar outcome trend is achieved for the testing phase by observing the sensitivity, specificity, precision, f-measure and accuracy value of $97.72 \%, 97.54 \%, 98.36 \%, 96.59 \%$ and $97.65 \%$ respectively with corresponding minimum error and loss values of 0.209 and 0.190 . The optimal and effective outcomes are achieved by the proposed methodology in terms of all the assessment indicators. This work specifically contributed in providing reliable outcomes for unfavorable real time industrial environment. The perspective of this work is that in future the direct object recognition capabilities will be explored in this research in order to provide larger degree of freedom for different types of robot arms. Moreover, the practicability of the research will be established by using reinforced learning for much complex environments.

\section{REFERENCES}

[1] Dahir, M. A., Obaid, A., Ali, A., Mohammed, A., Abou-ElNour, A., And Tarique, M., Mobile Based Robotic Wireless Path Controller, Netw. Protoc. Algorithms, 8(2), 20-38, 2016.

[2] MiEs, G., Military robots of the present and the future, Technology, 9(1), 125-137, 2010.

[3] Voth, D., A new generation of military robots, IEEE Intelligent Systems, 19(4), 2-3, 2004.

[4] Madhavan, R., Robots in military and aerospace technologies [news and views], IEEE Robotics \& Automation Magazine, $17(2), 1-6,2010$

[5] Shukla, A., AND Karki, H., A review of robotics in onshore oil-gas industry, In 2013 IEEE International Conference on Mechatronics and Automation, 1153-1160, 2013.

[6] Stavinoha, S., Chen, H., Walker, M., Zhang, B., And Fuhlbrigge, T., Challenges of robotics and automation in offshore oil and gas industry, In The 4th Annual IEEE International Conference on Cyber Technology in Automation, Control and Intelligent, 557-562, 2014.

[7] Anisi, D. A., Gunnar, J., Lillehagen, T., And Skourup, C., Robot automation in oil and gas facilities: Indoor and onsite demonstrations, In 2010 IEEE/RSJ International Conference on Intelligent Robots and Systems, 4729-4734, 2010.

[8] AQian, L., Zhang, X., Deguet, A., And Kazanzides, P., Aramis: Augmented reality assistance for minimally invasive surgery using a head-mounted display, In International Conference on Medical Image Computing and Computer-Assisted Intervention, 74-82, 2019.

[9] Mohareri, O., Schneider, C., Adebar, T. K., Yip, M. C., Black, P., Nguan, C. Y., ... And Salcudean, S. E., Ultrasound-based image guidance for robot-assisted laparoscopic radical prostatectomy: initial in-vivo results, In International Conference on Information Processing in Computer-Assisted Interventions, 40-50. Springer, Berlin, Heidelberg, 2013.

[10] Qian, L., Deguet, A., And Kazanzides, P., ARssist: augmented reality on a head-mounted display for the first assistant in robotic surgery, Healthcare technology letters, 5(5), 194-200, 2018.

[11] Qian, L., Song, C., Jiang, Y., Luo, Q., Ma, X., Chiu, P. W., ... and Kazanzides, P., FlexiVision: Teleporting the Surgeonâ $€^{T M}$ s Eyes via Robotic Flexible Endoscope and Head-Mounted Display, In IEEE/RSJ International Conference on Intelligent Robots and Systems (IROS), 2020.

[12] Karaman, S., And Frazzoli, E., Sampling-based algorithms for optimal motion planning, The international journal of robotics research, 30(7), 846-894, 2011.

[13] Spong, M. W., Hutchinson, S., And Vidyasagar, M., Robot modeling and control,3,75-118. New York: wiley, 2006.

[14] Qian, L., Wu, J. Y., DiMaio, S. P., Navab, N., and Kazanzides, P., A review of augmented reality in robotic-assisted surgery, IEEE Transactions on Medical Robotics and Bionics, 2(1), 1-16, 2019.

[15] Han, J., AND SEO, Y., Mobile robot path planning with surrounding point set and path improvement, Applied Soft Computing, $57,35-47,2017$.

[16] Huang, B., Timmons, N. G., And Li, Q., Augmented reality with multi-view merging for robot teleoperation, In Companion of the 2020 ACM/IEEE International Conference on Human-Robot Interaction, 260-262, 2020.

[17] Qureshi, A. H., And Ayaz, Y., Potential functions based sampling heuristic for optimal path planning, Autonomous Robots, 40(6), 1079-1093, 2016.

[18] He, Y., Fukuda, O., Ide, S., Okumura, H., Yamaguchi, N., and Bu, N., Simulation system for myoelectric hand prosthesis using augmented reality, In 2017 IEEE International Conference on Robotics and Biomimetics (ROBIO), $1424-1429,2017$.

[19] Yeole, A. R., Bramhankar, S. M., Wani, M. D., and Mahajan, M. P., Smart phone controlled robot using ATMEGA328 microcontroller, International Journal of Innovative Research in Computer and Communication Engineering, 3(1), 352$356,2015$. 
[20] Saquib, S. M. T., Hameed, S., Ali, S. M. U., Jafri, R., and Amin, I., Wireless Control of Miniaturized Mobile Vehicle for Indoor Surveillance, In IOP Conference Series: Materials Science and Engineering, 51(1), 012025. IOP Publishing, 2013.

[21] Bernhardt, S., Nicolau, S. A., Soler, L., and Doignon, C., The status of augmented reality in laparoscopic surgery as of 2016, Medical image analysis, 37, 66-90, 2017.

[22] Madhavan, K., Kolcun, J. P. G., Chieng, L. O., and Wang, M. Y., Augmented-reality integrated robotics in neurosurgery: are we there yet?, Neurosurgical focus, 42(5), E3, 2017.

[23] Qian, L., Deguet, A., Wang, Z., Liu, Y. H., and Kazanzides, P., Augmented reality assisted instrument insertion and tool manipulation for the first assistant in robotic surgery, In 2019 International Conference on Robotics and Automation (ICRA), 5173-5179, 2019.

[24] Hanna, M. G., Ahmed, I., Nine, J., Prajapati, S., and Pantanowitz, L., Augmented reality technology using Microsoft HoloLens in anatomic pathology, Archives of pathology \& laboratory medicine, 142(5), 638-644, 2018.

[25] Quero, G., Lapergola, A., Soler, L., Shahbaz, M., Hostettler, A., Collins, T., ... and Pessaux, P., Virtual and augmented reality in oncologic liver surgery, Surgical Oncology Clinics, 28(1), 31-44, 2019.

[26] Samei, G., Goksel, O., Lobo, J., Mohareri, O., Black, P., Rohling, R., and Salcudean, S., Real-time Fem-based registration of 3-D to 2.5-D transrectal ultrasound images,IEEE transactions on medical imaging, 37(8), 1877-1886, 2018.

[27] Lee, D., Kong, H. J., Kim, D., Yi, J. W., Chai, Y. J., Lee, K. E., and Kim, H. C., Preliminary study on application of augmented reality visualization in robotic thyroid surgery, Annals of surgical treatment and research, 95(6), 297, 2018.

[28] MourTzis, D., ). Simulation in the design and operation of manufacturing systems: state of the art and new trends, International Journal of Production Research, 58(7), 1927-1949, 2020.

[29] Mourtzis, D., Zogopoulos, V., And Xanthi, F., Augmented reality application to support the assembly of highly customized products and to adapt to production re-scheduling, The International Journal of Advanced Manufacturing Technology, 105(9), 3899-3910, 2019.

[30] Mourtzis, D., Samothrakis, V., Zogopoulos, V., and Vlachou, E., Warehouse design and operation using augmented reality technology: A papermaking industry case study, Procedia Cirp, 79, 574-579, 2019.

[31] Ong, S. K., Yew, A. W. W., Thanigaivel, N. K., and Nee, A. Y. C., Augmented reality-assisted robot programming system for industrial applications, Robotics and Computer-Integrated Manufacturing, 61, 101820, 2020.

[32] Ong, S. K., Nee, A. Y. C., Yew, A. W. W., And Thanigaivel, N. K., AR-assisted robot welding programming, Advances in Manufacturing, 8(1), 40-48, 2020.

[33] Avalle, G., De Pace, F., Fornaro, C., Manuri, F., and Sanna, A., AAn augmented reality system to support fault visualization in industrial robotic tasks, IEEE Access, 7, 132343-132359, 2019.

[34] Gong, L. L., Ong, S. K., And Nee, A. Y. C., Projection-based augmented reality interface for robot grasping tasks, In Proceedings of the 2019 4th International Conference on Robotics, Control and Automation, 100-104, 2019.

[35] Kitaev, N., Mordatch, I., Patil, S., and Abbeel, P., Physics-based trajectory optimization for grasping in cluttered environments, In 2015 IEEE International Conference on Robotics and Automation (ICRA), 3102-3109, 2015.

[36] Kopicki, M., Zurek, S., Stolkin, R., Moerwald, T., and Wyatt, J. L., Learning modular and transferable forward models of the motions of push manipulated objects, Autonomous Robots, 41(5), 1061-1082, 2017.

[37] Wei, Z., Chen, W., Wang, H., And Wang, J., Manipulator motion planning using flexible obstacle avoidance based on model learning, International Journal of Advanced Robotic Systems, 14(3), 1729881417703930, 2017.

Edited by: Pradeep Kumar Singh

Received: May 31, 2021

Accepted: Sep 13, 2021 
